\title{
Roughness Perception in Virtual Textures
}

\author{
Bertram Unger, Member, IEEE, Ralph Hollis, Fellow, IEEE, and Roberta Klatzky
}

\begin{abstract}
Haptic devices allow the production of virtual textured surfaces for psychophysical experiments. Some studies have shown inconsistencies between virtual and real textures with respect to their psychophysical functions for roughness, leading to speculation that virtual textures differ in some way from real ones. We have determined the psychophysical function for roughness using textures rendered with a high-fidelity magnetic levitation haptic device. A constraint surface algorithm was used to simulate the motion of a spherical probe over trapezoidal gratings and randomly dithered cones. The shape of the psychophysical functions for roughness is consistent between subjects but varies with changes in texture and probe geometry. For dithered cones, inverted "U"-shaped functions were found nearly identical, in maxima and curvature, to those in the literature for real textures with similar geometry.
\end{abstract}

Index Terms-Haptics, Psychophysics, Texture, Roughness, Perception.

\section{INTRODUCTION}

$\mathrm{T}$ $O$ understand the perceptual processes involved in perceiving texture by touch, a common approach relies on determining the relationship between a physical factor in the environment and a quantifiable measure of the perception of that factor as texture. The perceived textural dimension most commonly studied is roughness [1], [2], [3], [4], [5], [6], [7], [8], [9], [10], [11], [12], since it has been found to be systematically related to a number of physical properties.

A carefully controlled analysis of the relationship between physical stimuli and roughness was conducted by Lederman [6]. The experiments, carried out with a bare finger on a set of manufactured gratings, demonstrated that perceived roughness was directly related to the spatial deformation of the fingertip's skin by the textured grooves [6], [11], [13], [14], [15]. The velocity of the finger during active or passive exploration [6], [8], [11] had no effect, further implicating a spatial code. Other supporting findings come from neurophysiological studies that measure the output of the mechanoreceptors present in the fingertip skin of primates in relation to perceived roughness [2], [16], [17], [18], [19], [20]. Temporal coding is also disconfirmed by the finding that selective adaptation of receptors to vibration results in little change in perceived roughness [14].

Recent studies indicate that while spatial effects predominate with bare-finger texture perception, vibrational and temporal cues may provide an alternative channel of information [1], [21]. While selective adaptation of vibrational receptors (FA1's and FA2's) does not preclude roughness perception, it does produce changes in its quality [22], [23]. Hollins et al. developed a duplex theory

- Bertram Unger is with the Faculty of Medicine at the University of Manitoba, (contact: ungerb@cc.umanitoba.ca).

- Ralph Hollis is with the Robotics Institute at Carnegie Mellon University (contact: rhollis@cs.cmu.edu).

- Roberta Klatzky is with the Department of Psychology at Carnegie Mellon University. (contact: klatzky@andrew.cmu.edu). of texture perception [23], [24], [25] demonstrating that the roughness of small period textures $(<1 \mathrm{~mm})$ may be mediated by vibration, while that of larger periods may be mediated by spatial cues.

The interposition of a rigid link, such as a probe, between surface texture and the user removes the possibility that spatial effects can mediate roughness perception. Nevertheless, studies demonstrate clearly that roughness is well differentiated when textured surfaces are explored with a probe [5], [26], [27]. These findings suggest that in the absence of spatial information, vibrational information can underlie roughness perception. The role of vibration is supported by the fact that roughness estimates using a probe are affected by changes in probe speed [26], [28], in contrast to Lederman's work with the bare finger [14].

There is, however, a difference in the psychophysical function relating judgments of roughness magnitude to the physical structure of a textured surface when surfaces are felt with a probe, in comparison to the bare finger. Klatzky and associates found that as the spacing between elements on a surface increased, so too did subjects' estimates of surface roughness, rising to a maximum and then falling off [26], [28]. The resulting roughly quadratic shape of perceived roughness intensity versus element spacing was attributed to the geometric relationship between probe and elements. Based on these data, Klatzky et al. developed a geometric model of probe-texture interaction. This model predicts, based on texture geometry, the effect of changing probe size on roughness intensity [28]. In the case of a non-point probe with real physical size, when element spacings are small with respect to the probe tip, the probe travels along the tops of the elements, penetrating only slightly, if at all (see Fig. 1, bottom). The forces transmitted by the probe to the finger will vary only slightly. As element spacing increases, the probe tip begins to penetrate more deeply between gratings and, for a given probe speed, force variation will increase. At a spacing where the probe penetrates to maximum depth and follows the texture 
surface precisely (referred to by Klatzky et al. as the droppoint (DP); see Fig. 1, top panel), roughness intensity should be maximal. Further increases in element spacing now result in decreases in force variation and thus lower perceived roughness intensity. The geometric model's
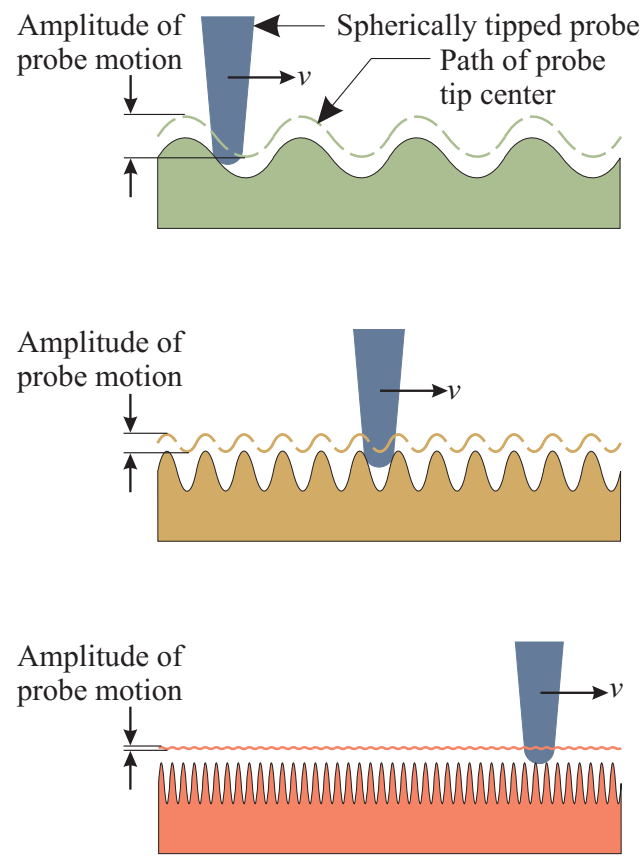

Fig. 1. The effect of texture spatial frequency on probe motion amplitude.

predictions were experimentally verified by Klatzky et al. When a spherical probe was used to explore textures with varying inter-element spacings, the psychophysical function relating roughness to spacing was an inverted "U" that was roughly quadratic in shape. This curve had a maximum near the predicted element spacing [28].

Haptic devices have created new possibilities for the study of texture perception. Haptic simulations of texture vary the position and/or forces applied to the hand while users move a probe across a textured surface. The parameters can be accurately and rapidly changed, allowing researchers to investigate human perceptual responses to a wide range of textures. Although haptic textures can be rapidly simulated at less cost than their physical counterparts, serious questions remain about their validity. Do they accurately represent physical textures? Does simulation introduce significant or subtle changes in the perceptual quality of the texture? Is the human psychological response the same for simulated and real textures?

Interestingly, initial attempts to mimic psychophysical data by using haptic simulations of probes exploring gratings have not been successful. Studies demonstrate that as groove width (and thus element period) increases, roughness perception usually decreases in a linear fashion, although individual fits may vary [4], [7], [9], [29]. The quadratic roughness perception function of Klatzky et al.'s studies on real textures, which was reliably ob- served across individual subjects, is not seen in these simulations. The source of the discrepancy is not clear; it may be related to limitations in the haptic system itself or to simplifications and assumptions made by the texture simulation model.

Penn et al. speculated that the decline in roughness perception experienced by subjects in studies employing sinusoidal virtual textures is due to the infinitesimal nature of the haptic interaction point (HIP) used in the haptic model [9]. Given its size, the HIP follows the sinusoid exactly, regardless of period, and thus shorter periods may result in higher-frequency vibrations and increased changes in force along the direction of the probe axis. Since human roughness estimations have been shown to be correlated with the rate of change of tangential force on the finger pad during direct touch, this may explain the decrease in perceived roughness with increasing wavelength seen in virtual-texture studies [10].

In this paper we employ a high-fidelity haptic device to generate a variety of virtual textures and investigate their subjective roughness. We first examine sinusoidal grating textures explored with an infinitely small virtual point probe. Unlike previous studies, we find a consistent psychophysical function relating sinusoidal wavelength to perceived roughness. We then consider the interaction of probe and texture geometry and develop a trapezoidal grating texture which can be explored with a spherical probe. Perceived roughness for these textures exhibits a substantially different psychophysical function, lending support to the idea that the virtual model's characteristics play a large role in determining its perceived roughness while hardware considerations may be less important. Finally, we demonstrate that, by careful simulation of texture and probe geometry, roughness perception of virtual surfaces, as measured by the psychophysical function, can be essentially equivalent to that of real surfaces.

\section{Experimental Setup}

\subsection{Magnetic Levitation Haptic Device}

Our experiment employs a 6-DOF magnetic levitation haptic device (MLHD) using Lorentz forces [30], [31], [32]. Performance characteristics are given in Table 1.

The system is capable of rendering virtual textures with high fidelity. A proportional-derivative (PD) controller running on an AMD 2100+ processor controlled the device with a servo update rate of $1000 \mathrm{~Hz}$. Proportional gains were set to nominal values of $10 \mathrm{~N} / \mathrm{mm}$ in translation and $25 \mathrm{Nm} /$ radian for orientation. Derivative gains were set to $0.04 \mathrm{~N} / \mathrm{mm} / \mathrm{s}$ in translation and $0.5 \mathrm{~N} / \mathrm{radian} / \mathrm{s}$ for orientation. Only $z$-axis forces were actively generated by a penetration depth algorithm and rotation was not permitted. The device inherently has no static friction and static friction was not simulated. A feedforward force was applied, reducing the force of gravity on the manipulandum (see Fig. 2). 


\begin{tabular}{|l|l|}
\hline Parameter & Value \\
\hline \hline Translational range & $25 \mathrm{~mm}$ \\
Rotational range & $15-20^{\circ}$ \\
Motion resolution & $5-10 \mu \mathrm{m}$ \\
Perceived mass & $580 \mathrm{~g}$ \\
Perceived inertia & $0.0054 \mathrm{~kg} / \mathrm{m}^{2}$ \\
Maximum force & $>55 \mathrm{~N}$ \\
Maximum torque & $>6.3 \mathrm{Nm}$ \\
Maximum stiffness & $25 \mathrm{~N} / \mathrm{mm}$ \\
Minimum stiffness & $.005 \mathrm{~N} / \mathrm{mm}$ \\
Position bandwidth & $120 \mathrm{~Hz}$ \\
\hline
\end{tabular}

TABLE 1

Performance characteristics of the magnetic levitation haptic device used in the experiments.

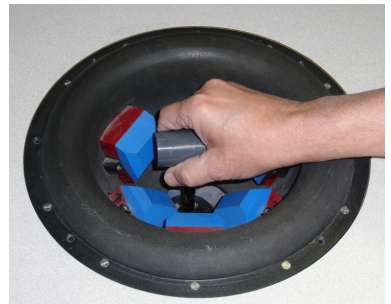

Fig. 2. Subject's hand on the manipulandum of the magnetic levitation haptic device.

\subsection{Texture Simulation Algorithms}

We conducted three experiments using three different virtual textures: a sinusoidal grating texture (SGT), a trapezoidal grating texture (TGT) and a dithered conical texture (DCT) of truncated cone-shaped elements.

The SGT is the simplest of the simulations; it maps the HIP as an infinitely small probe. When the probe is in contact with the surface, a force is generated proportional and opposed to its penetration depth into a virtual sinusoidal surface along the $z$ axis. The orientation of the manipulandum is controlled to keep it vertical at all times. When the probe is not in contact with the surface it is allowed to fly freely, experiencing no forces other than gravity.

In order to explore the interaction of probe and texture geometry a more sophisticated algorithm was developed. The probe is no longer considered to be a point and is given size and shape. Changing the probe geometry from an infinitely small point to an object that has some shape means that the HIP at the probe center can no longer follow texture contours exactly, but instead must follow a surface determined by the interaction of probe shape and texture geometry, as seen in Figure 3. This surface is used to control the position of the HIP as if it were at the center of a probe with some substantive shape (a spherical case is shown in Fig. 3). We refer to the surface followed by the HIP under these conditions as the constraint surface (CS).

The CS is composed of groupings of all the points for which the same motion constraints apply, a concept well known from the motion planning literature [33]. In practice, a set of parametric equations is used to indicate

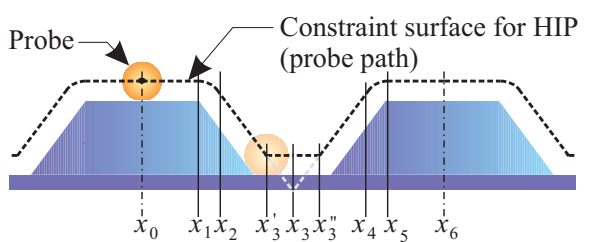

(a)

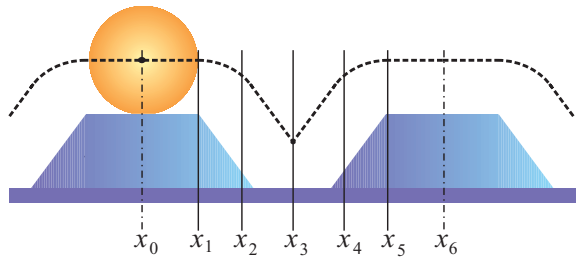

(b)

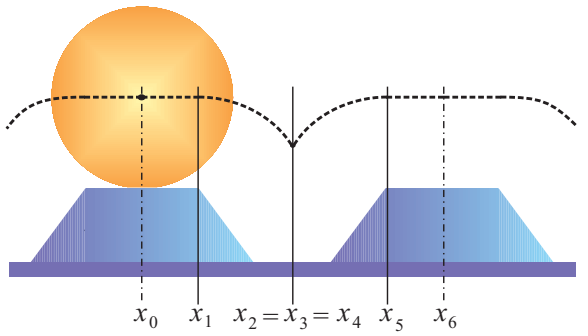

(c)

Fig. 3. Cross-section of trapezoidal grating texture with inflection points: (a) small probe, $(b)$ larger probe, $(c)$ very large probe.

the desired height, $z_{d e s}$, of the HIP above the base plane of the texture (located at 0 on the $z$ axis) given its current $x$ and $y$ coordinates (see Appendix A).

During initialization of a texture, the $(x, y)$ location of each element is stored in computer memory. Using the coordinates of the HIP in the physical device workspace as an index into memory, the algorithm determines the nearest element to the HIP. The distance from the HIP to the element determines the parametric equation to be used in the calculation of the height of the CS at that point, $z_{\text {des }}$. The difference between the desired probe height $z_{d e s}$, and the actual location of the point $z_{a c t}$, is the error signal $z_{e r r}$. The $z_{e r r}$ value is used by a PD controller running at $1000 \mathrm{~Hz}$ to calculate the output force required to drive the position error to zero. Haptic textures are thus generated from the CS model as the MLHD manipulandum is moved through the device workspace. As a subject moves about the $(x, y)$ plane, the manipulandum moves up and down vertically, its height depending on the geometry of the probe and the texture which is modeled beneath it. Given the geometry of the texture elements (described below), the update rate, and subjects' typical exploration speed, this algorithm produces highly stable textures.

It is important to note that the CS model, as formulated, is a quasistatic model which assumes that motions between control cycles will be very small. Under its assumptions, the probe is regarded as effectively stationary at any given instant in time, and dynamics are assumed to play no role in its behavior. 
Two different constraint surfaces are used in our experiments. The first CS is based on the interaction of a spherical probe with a TGT. This texture, with its sharp inflection points, provides a significant departure from the smoothly curved SGTs commonly used in other studies [4], [7], [9], [29]. The second CS derives from a spherical probe and pseudo-randomly distributed truncated conical elements. This DCT surface is intended to compare closely with real textures used by Klatzky et al. in psychophysical studies of indirect touch [28].

\subsection{General Experimental Design and Procedure}

Roughness magnitude estimation experiments were performed by subjects from a student pool associated with the psychology department at Carnegie Mellon University, who received credit for participation. Additional subjects were paid and unpaid student volunteers from other departments and from within the Microdynamics Systems Laboratory at Carnegie Mellon. All experiments were performed with IRB approval.

Subjects were seated approximately $500 \mathrm{~mm}$ from a graphical display. Textures were presented haptically to the user's right-hand fingertips and palm by the MLHD manipulandum. Subjects could not see the texture and listened to white noise via headphones to prevent auditory identification of texture roughness.

Subjects were informed that they would be presented with a variety of textures to explore with the MLHD manipulandum. No restrictions were placed on exploration other than a warning that excessive force would cause the device to shut down. After exploration, they gave an estimate of the roughness magnitude of the explored surface by entering a number on a computer keypad. Instructions indicated that larger numbers were to reflect larger values of roughness, and that zero or negative numbers should not be used. In addition to the magnitude response, MLHD manipulandum position and force data were recorded throughout the experiment at a $1000 \mathrm{~Hz}$ sampling frequency. Trials representing each combination of a texture and probe were presented to subjects a number, $n_{\text {iterate }}$, of times, to reduce variability in the magnitude estimates. If there were $n_{\text {space }}$ element spacings and $n_{\text {size }}$ probe sizes, then a subject explored $n_{\text {trials }}=n_{\text {iterate }} n_{\text {space }} n_{\text {size }}$ texture surfaces in the course of the experiment. A preliminary demonstration block was included, representing the range of texture to be experienced. The order of texture-probe pairings was randomized within both demonstration and experimental blocks.

\subsection{Determining the Psychophysical Function}

We use the psychophysical function, $S=\Psi(I)$, to assess the effects of geometry on roughness perception. The process for determining $S=\Psi(I)$ uses subjects' numeric responses to estimate their roughness perception, $S$, of a stimulus having an inter-element spacing of texture elements, $I$. If there are $k=1, \ldots, n_{\text {space }}$ different interelement spacings, a single experimental trial consists of a subject's numerical estimate of the roughness of the kth texture spacing and $S_{k}=\Psi\left(I_{k}\right)$. Multiple iterations of each trial are performed and averaged to obtain a single data point in the subject's psychophysical function for roughness, $\bar{S}_{k}=\bar{\Psi}\left(I_{k}\right)$. Outlier estimate values more than 10 times the median of a subject's estimates were considered to be due to subject data entry error and were excluded from the calculations.

If there are $i=1, \ldots, n_{s u b j}$ subjects in an experiment, a single subject's psychophysical profile, $S_{i}=\Psi\left(I_{i}\right)$, can then be described as the set

$$
S_{i}=\left\{\bar{S}_{1}, \bar{S}_{2}, \ldots, \bar{S}_{n_{\text {subj }}}\right\} .
$$

Because each subject chose his or her own magnitude estimation scale with which to represent roughness, it was necessary to normalize the $S_{i}=\Psi\left(I_{i}\right)$ values for purposes of comparison. The normalized percept, $\hat{S}_{i}$ was obtained by dividing $S_{i}$ by the mean of all estimates by subject $i$, and multiplying by the mean of all estimates by all subjects in the experiment. The normalized means for a given probe size can then be averaged across all subjects in the experiment to yield a cross-subject, normalized mean perception, $\bar{S}=\Psi(I)$. In the remainder of this paper we will simply use $\Psi(I)$ to refer to the normalized individual-subject function or the averaged function, as appropriate in context.

\section{Sinusoidal Grating Texture Experi- MENT}

\subsection{Experimental Design}

Virtual SGTs (see Fig. 4) with spatial periods ranging from 0.025 to $6.00 \mathrm{~mm}$ were generated using the MLHD. A penetration depth algorithm was used. The smallest grating periods approached the resolution of the MLHD. The largest grating periods allowed 4 spatial periods within the MLHD's workspace. Thirty-three different periods were used. The period space was sampled asymmetrically, with a larger number of samples from the shorter periods. The sinusoid amplitude was 0.4 $\mathrm{mm}$ peak-to-peak, consistent with the height of texture elements investigated by other studies [28].

The experiment used an infinitely small point-probe. A demonstration block (33 trials) was followed by experimental trials consisting of 33 textures, presented 4 times each in random order, for a total of 132 recorded trials.

\subsection{Results and Discussion}

Magnitude estimation data were collected for 27 subjects. The psychophysical function $\hat{S}_{i}=\Psi\left(I_{i}\right)$ was calculated for each subject and the overall psychophysical function $\bar{S}=\Psi(I)$ was found by averaging the normalized individual functions, following the techniques outlined in Section 2.4. 


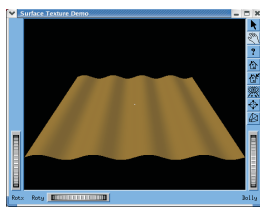

(a)

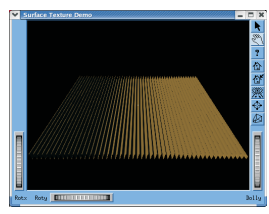

(c)

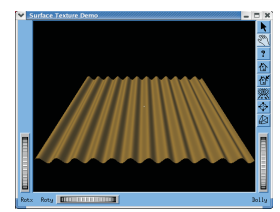

(b)

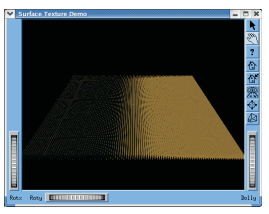

(d)
Fig. 4. Graphic representation of four examples of SGTs used for psychophysical experiments with a (a) $6 \mathrm{~mm}$ period, (b) 2 $\mathrm{mm}$ period, $(c) 0.50 \mathrm{~mm}$ grating and $(d) 0.25 \mathrm{~mm}$ grating.

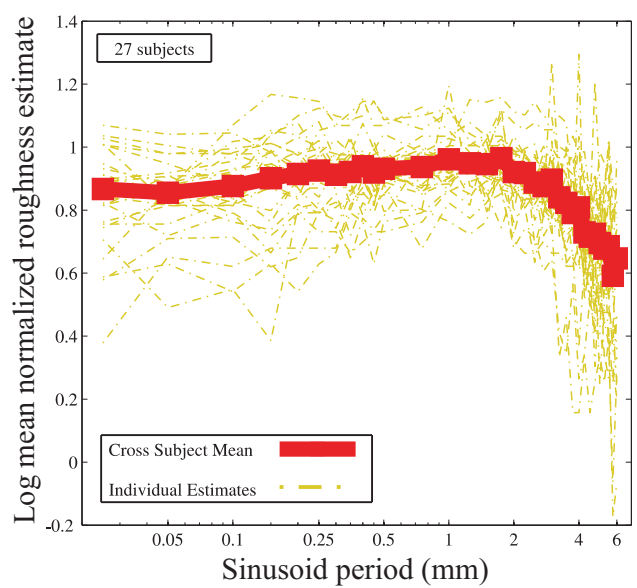

Fig. 5. Log-log plot of individual normalized roughness psychophysical functions for 27 subjects superimposed on their cross subject mean for SGTs.

Superimposed log-log plots of the normalized $\Psi\left(I_{i}\right)$ for each subject as well as the mean roughness function can be seen in Fig. 5. Although the functions show considerable variance between individual subjects, most follow a pattern of an initial rise followed by a decline in roughness as a function of increasing texture period. A one-way ANOVA showed that element spacing had a significant effect on roughness magnitude $(\mathrm{F}(32$, 726) $=11.52, \mathrm{p}<0.0001$ ).

The mean roughness function changes in slope from positive to negative at an element spacing of approximately $2 \mathrm{~mm}$. The first part of the function, where periods are less than $2 \mathrm{~mm}$, is flat and has a mean log-log slope close to zero (see Table 2). The overall slope for periods of greater than $2 \mathrm{~mm}$ is approximately -0.8 , virtually identical to the log-log slope (Stevens' power law exponent $b$ ) determined by others for similar surfaces [4].

The reasons for the bipartite behavior of roughness estimates on SGTs is unclear. One possibility is that the magnitude of the as yet undetermined physical property underlying the roughness of SGTs is relatively constant below a $2 \mathrm{~mm}$ period but declines above $2 \mathrm{~mm}$. In

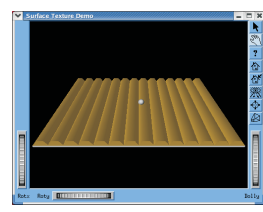

(a)

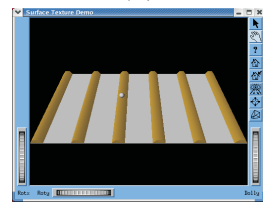

(c)

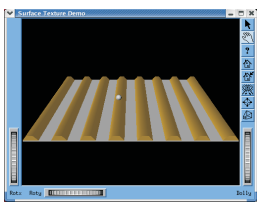

(b)

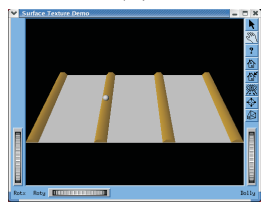

(d)
Fig. 6. Graphic representation of four examples of TGT used for psychophysical experiments with a (a) $0.96 \mathrm{~mm}$ period, (b) $1.96 \mathrm{~mm}$ period, (c) $3.46 \mathrm{~mm}$ grating and (d) $5.96 \mathrm{~mm}$ grating.

this case the magnitude of this physical property should correlate with roughness perception; it should show a flat or slightly rising slope below a $2 \mathrm{~mm}$ period and a more steep, descending slope above it. A second explanation is that the bipartite behavior is a function of the underlying neural processing. For example, it is possible that sinusoidal gratings spaced more closely than $2 \mathrm{~mm}$ create vibrations that are below the threshold of the operative neuroreceptors or elicit a constant receptor response. Finally, device or algorithm limitations may limit rendering for smaller texture spacings.

\section{Trapezoidal Grating Texture Experi- MENT}

\subsection{Experimental Design}

Virtual TGTs similar in height, width and side angle (see Figure 6) to Klatzky et al.'s fabricated truncated cone textures [28] were generated using the CS algorithm. Elements were $0.42 \mathrm{~mm}$ high with a plateau width of $0.46 \mathrm{~mm}$ and a base width of $1.04 \mathrm{~mm}$. These dimensions result in an element side angle, $\alpha$, of $53^{\circ}$. Element spacings ranged from $0.96-5.96 \mathrm{~mm}$. Four probe radii $(0.25$, $0.5,1.0$ and $1.5 \mathrm{~mm}$ ) were used, allowing comparison with Klatzky et al.'s physical texture studies where probe radii ranged from $0.5-1.5 \mathrm{~mm}$ [28].

Nine female and 17 male subjects, including 3 lefthanded, performed the experiment. All subjects used the right hand.

An experimental trial consisted of a subject exploring a single texture period with a single probe radius. Four probe sizes, eleven texture periods and 3 iterations produced 132 trials per subject. The experiment was blocked by probe size, with 3 repetitions of each texture period randomly ordered within a block. Each block began with six demonstration trials using that block's probe size. Block order was randomized between subjects to prevent learning effects.

\subsection{Results}

The psychophysical function for roughness perception, $\Psi(I)$, was obtained for each subject and the mean over 


\begin{tabular}{|c|c|c|c|c|c|c|}
\hline Texture Period Range [mm] & Mean slope & Median & $\sigma$ & Maximum & Minimum & \# Positive/Negative \\
\hline $0.025-1.75$ & 0.067 & 0.041 & 0.118 & 0.328 & -0.160 & $20 / 7$ \\
$2.25-6.0$ & -0.823 & -0.703 & 0.676 & 0.084 & -2.775 & $2 / 25$ \\
\hline
\end{tabular}

TABLE 2

Slope of roughness function in a log-log plot for small SGT periods $(0.025-1.75 \mathrm{~mm})$ and large SGT periods $(2.25-6.0 \mathrm{~mm})$.

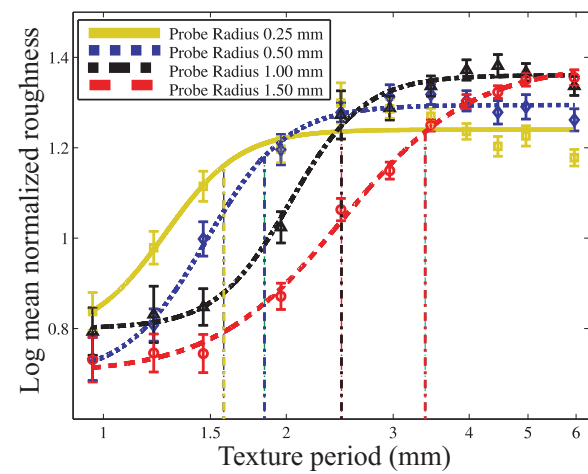

Fig. 7. Unconstrained nonlinear optimization fit of logistic curve to mean normalized roughness estimates for TGTs explored with spherical probes of $0.25,0.5,1.0$ and $1.5 \mathrm{~mm}$ radius. Vertical lines indicate $80 \%$ of height of each curve.

subjects for each probe size, using the averaging and normalization techniques described in Section 2.4. The mean psychophysical function data could be fit reasonably well with a second order quadratic or third order polynomial, with $R^{2}$ values for a second order fit ranging from 0.93-0.98 across the probe conditions.

Concerning the individual psychophysical functions, a quadratic fit had a mean $R^{2}$ value of 0.85 across subjects and probe types, while the linear fit had a mean $R^{2}$ of 0.69. Clearly, individual subjects do not perceive texture as a linear function of element spacing for TGTs, and the quadratic trend seen in the cross-subject aggregate data seems consistent with the individual data.

\subsection{Discussion}

Examining the maxima of the psychophysical functions seen in Table 3, it can be observed that, for both second and third order fits, maxima move to larger texture periods as probe size increases. This behavior is predicted by the geometric model of probe and texture interaction, developed for real textures and probes by Klatzky et al. [28]. Intuitively, this is because larger probes require larger element spacings before they can fully penetrate between grating elements. The predicted location of the psychophysical function maxima for the four probe sizes used in the experiment can also be seen in Table 3. It should be noted that the location of the fitted function maximum represents an experimentally determined approximation of the DP. A DP is, by definition, calculated from the geometry of the probe-texture interaction [28].

Although Table 3 shows that, regardless of the kind of fit chosen for the roughness function data, the DP increases with element spacing, it also reveals that both second and third order fits tend to produce maxima which are underestimated by the geometric model. A 1way ANOVA for the effects of probe size on DP showed significance only for the third order fit and not the second (Table 4). This may be due to the fact that the actual data do not really appear to be well fit by a polynomial function. Rather, a step function, such as a sigmoidal logistic curve, seems a more likely fit.

Figure 7 shows an unconstrained nonlinear optimization fit of a logistic curve of the form:

$$
y=C+\frac{A}{1+e^{-\lambda(x-B)}}
$$

to the averaged and normalized roughness perception data. The sums of squared errors are very low $(0.013$, $0.004,0.004$ and 0.002 for probe sizes of $0.25,0.5,1.0$ and $1.5 \mathrm{~mm}$ respectively), and the function appears to fit the shape of the data well. The point at which the logistic curve nears its maximum could be considered the point of maximum roughness or the equivalent of the DP. Arbitrarily choosing $80 \%$ of a logistic curve's $y$-axis range as a measure of the DP, it can be seen that for a logistic fit, the DP also increases with element spacing (Table 3). The DP's from the logistic curve are still generally larger than the predicted values.

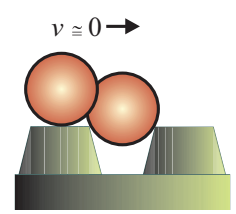

(a)

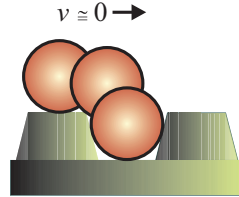

(b)

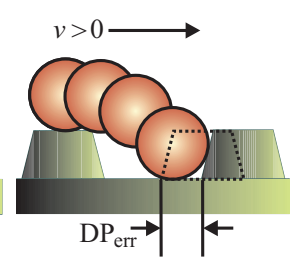

(c)
Fig. 8. Drop point (DP) error explained by probe velocity and geometric model: in (a) and (b) when velocity is 0 , DP is determined only by geometry; in $(c)$ with velocity greater than 0 , $\mathrm{DP}$ is determined by probe velocity and geometry.

The underestimation of the DP by the geometric model may be due to its underlying quasi-static assumption. The model assumes that depth of penetration is only related to probe position relative to texture geometry; dynamic effects are ignored (Figs. 8a and b). In fact, the probe has some velocity that will carry it forward as it falls to the floor between elements. Element separation must therefore be slightly larger than that predicted by geometry alone, in order for a moving probe to reach the floor without contacting the next element's leading edge, as seen in Fig. 8c. A subject's hand and 


\begin{tabular}{|c|c|c|c|c|c|}
\hline Rad. & 2nd Ord. Curv. & Pred. Max $[\mathrm{mm}]$ & 2nd Ord. Max $[\mathrm{mm}]$ & 3rd Ord. Max [mm] & Logis. Max [mm] \\
\hline $0.25 \mathrm{~mm}$ & -1.65 & 1.31 & 3.15 & 3.00 & 1.58 \\
$0.50 \mathrm{~mm}$ & -1.77 & 1.56 & 3.79 & 3.79 & 1.84 \\
$1.0 \mathrm{~mm}$ & -0.85 & 2.06 & 7.75 & 4.43 & 2.47 \\
$1.5 \mathrm{~mm}$ & 0.077 & 2.56 & N/A & 5.30 & 3.39 \\
\hline
\end{tabular}

TABLE 3

The TGT period at which maximum roughness occurs, based on 2nd and 3rd order polynomial curves fitted to roughness estimates averaged across subjects as well as the height of a fitted logistic curve ( $80 \%$ of max-min). Curvature of the 2 nd order fitted curve (on a log-log plot) and the period of maximum roughness predicted by the geometric model are also shown.

\begin{tabular}{|c|c|c|c|}
\hline Factor & DOF & $\mathrm{F}$ & $\mathrm{p}$-value \\
\hline Curvature & $3 / 92$ & 23.47 & $<0.0001$ \\
2nd Order Maxima & $3 / 92$ & 0.52 & $>0.05$ \\
3rd Order Maxima & $3 / 92$ & 34.26 & $<0.0001$ \\
\hline
\end{tabular}

TABLE 4

Significance of probe size effects with TGTs, determined using a 1-way ANOVA on curvature and maxima of a 2nd and 3rd order fit to subject roughness estimates.

the manipulandum are subject to gravity resulting in a downward acceleration, $g$. An approximate idea of the underestimation in DP, $D P_{\text {err }}$, resulting from the model's quasi-static assumption, can thus be calculated from element height, $e l_{h}$, and the planar velocity, $v_{x y}$, of the probe.

$$
D P_{e r r}=v_{x y} \sqrt{\frac{2 e l_{h}}{g}} .
$$

Mean planar velocity (MPV) over all subjects, trials and probe sizes was determined from position recordings made directly from the MLHD and was found to be consistent with velocities used in real-texture studies [6], [28], [34] with small inter-subject variability. Using Equation 3 and the measured MPV value of $51.08 \pm 0.29$ $\mathrm{mm} / \mathrm{sec}$, a $D P_{\text {err }}$ of $0.47 \mathrm{~mm}$ was found. This value approaches the average measured $D P_{\text {err }}$ of $0.45 \mathrm{~mm}$ for the logistic fit to simulated TGTs and is close to the $0.24 \mathrm{~mm}$ average $D P_{\text {err }}$ found with real dithered textures [28]. In previous studies, the element spacing corresponding to the DP has been found to increase with probe velocity [27], [28]. This is consistent with the predictions of our new velocity-revised geometric model.

\section{Dithered Conical Texture Experi- MENT}

\subsection{Experimental Design}

The CS algorithm was used to generate a set of virtual DCTs (see Fig. 9). The size and shape of the conical texture elements was comparable to elements in Klatzky et al.'s studies of real textures [28] and in our second experiment. Electron micrographs of one of Klatzky et al.'s physical texture plates are shown in Fig. 10.

The present conical elements had a height, $C_{h}$, of $0.42 \mathrm{~mm}$ and side angle, $\alpha$, of $53^{\circ}$. The base and top radii $\left(R_{\text {base }}=0.52 \mathrm{~mm}\right.$ and $\left.R_{\text {top }}=0.23 \mathrm{~mm}\right)$ of the DCT elements were equal to half of the base and

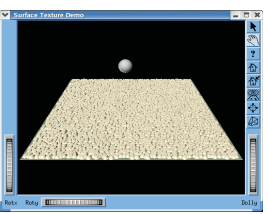

(a)

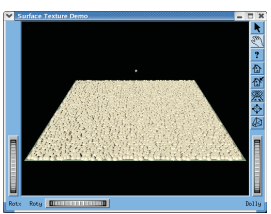

(c)

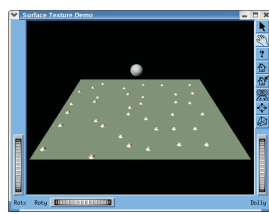

(b)

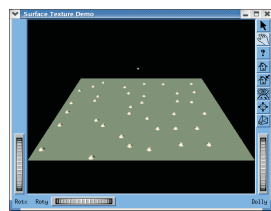

(d)
Fig. 9. Graphical representation of the range of DCT models. Textures have element spacings ranging from 0.5 to $5.5 \mathrm{~mm}$ and probe radii ranging from 0.25 to $1.5 \mathrm{~mm}$. (a) probe size $=1.5$ $\mathrm{mm}$, spacing $=0.5 \mathrm{~mm},(b)$ probe size $=1.5 \mathrm{~mm}$, spacing $=5.5$ $\mathrm{mm},(c)$ probe size $=0.25 \mathrm{~mm}$, spacing $=0.5 \mathrm{~mm},(d)$ probe size $=0.25 \mathrm{~mm}$, spacing $=5.5 \mathrm{~mm}$.

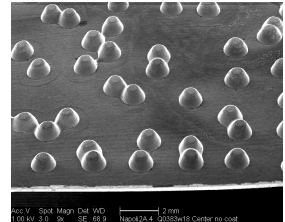

(a)

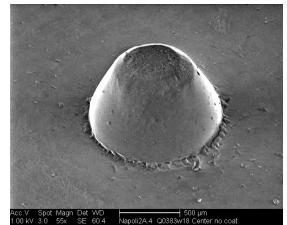

(b)
Fig. 10. Electron micrographs of Klatzky et al.'s texture plates [28]: (a) view of small area of plate, $(b)$ view of single cone.

plateau widths, respectively, of the previously used TGT elements. Element size was constant throughout the experiment.

Elements were laid out on a grid at their desired spacing and then randomly moved from their initial location by up to 40 percent of their initial spacing using zero-mean white noise. This resulted in a pseudorandom distribution which maintained the original mean 
spacing. Eleven element spacings (0.5, 0.75, 1.0, 1.5, 2.0, $2.5,3.0,3.5,4.0,4.5$ and $5.5 \mathrm{~mm}$ ) were used. This range was subject, at its upper end, to the limitations of the MLHD workspace, and at its lower end, to the MLHD's position bandwidth.

Four spherical probes with radii of $0.25,0.5,1.0$ and 1.5 $\mathrm{mm}$, were used. The probe sizes selected had predicted roughness maxima which fell within the selected range of texture spacings.

Four probe radii and 11 spacings generated 44 probespacing combinations, which were repeated 3 times each. In order to allow comparison of absolute roughness magnitude between probes, blocking was not used and the 132 trials were presented in random order. This should lead a subject to use the same internal roughness scale throughout the experiment. A preliminary demonstration block of 24 trials was presented, consisting of six representative texture spacings crossed with all four probe sizes.

Seven female and seventeen male subjects, for a total of 24 subjects, including 4 left-handed subjects, performed the experiment. All subjects used the right hand.

\subsection{Results}

A quadratic fit approximates the mean roughness magnitude estimation data well for the DCTs (see Fig. 11). Individual roughness perception functions are shown in Figure 12. They are quite variable but the majority of them are well fit by a quadratic curve.

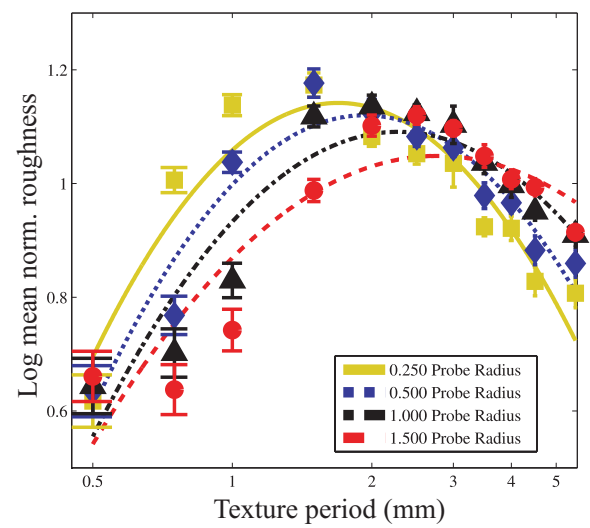

Fig. 11. Second order fit to mean normalized roughness estimates of DCTs explored with spherical probes.

The element spacings of maximum roughness predicted by Klatzky et al.'s geometric model [28], along with the locations of the maxima of second and third order fits to experimentally determined subjective roughness, can be seen in Table 5. A 1-way ANOVA showed a significant effect of probe radius on the maxima of both the second and third order fit as well as the second order curvature (see Table 6). The maximum subjective roughness reported by subjects was not significantly affected by probe radius.

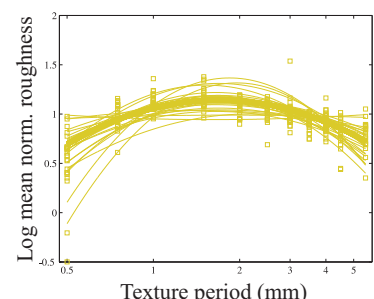

(a)

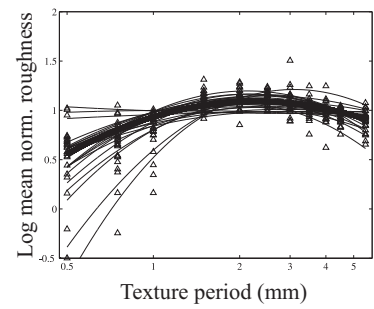

(c)

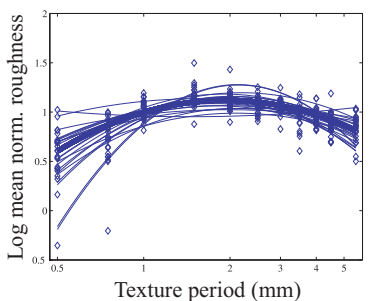

(b)

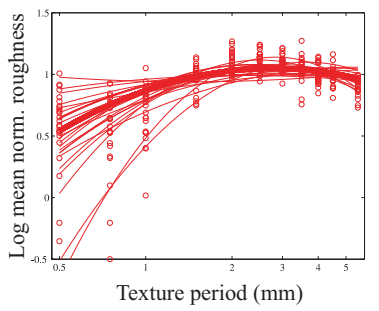

(d)
Fig. 12. Thin lines represent individual subject roughness estimates for DCTs fitted with 2nd order curves in a log-log plot. The second order fit to the mean data is plotted as a thick line. Each plot represents a single probe radius as follows: (a) 0.25 $\mathrm{mm},($ b) $0.50 \mathrm{~mm},($ c) $1.0 \mathrm{~mm}$, and (d) $1.5 \mathrm{~mm}$.

\subsection{Discussion}

The psychophysical functions found by individual subjects had fitted quadratics similar to those found by Klatzky et al. using real DCTs and spherical probes [28]. They bear little resemblance to the linear functions found for virtual sinusoidal textures in this paper or previous studies [4], [7], [9], [29]. Clearly, probe-texture geometry plays an important role in determining the shape of the psychophysical function for roughness. Increasing probe radius increased the spacing at which subjects reported maximum roughness, as predicted by the geometric model [28]. The underestimation of the point of maximum roughness by the model, can be explained once again with reference to the model's quasi-static nature (see Section 4.3). The average error $\left(D P_{e r r}\right)$ is $+0.30 \mathrm{~mm}$ for second order maxima and $+0.31 \mathrm{~mm}$ for the third order maxima. Correcting the $D P$ for a mean planar velocity of $49.06 \pm 3.34 \mathrm{~mm} / \mathrm{sec}$, and using Equation 3, the velocity-corrected $D P_{\text {err }}$ is $+0.45 \mathrm{~mm}$. The DP's for virtual DCTs are thus very close to the revised geometric model's predicted values.

The measured locations of virtual DCT DP's are also similar to those found for corresponding real textures. Klatzky et al. found maxima of 1.70, 2.23 and $2.98 \mathrm{~mm}$ for probes with radii of $0.5,1.0$, and $1.5 \mathrm{~mm}$ respectively [28]. These values are corrected to reflect spacings taken from element center to element center, as opposed to the so-called HV spacing between element edges used by Klatzky et al. This allows direct comparison to our results, as seen in Figure 13. The mean difference between Klatzky et al.'s real roughness $\Psi(I)$ maxima and the geometric model's predictions, is $+0.24 \mathrm{~mm}$. The mean difference between Klatzky et al.'s real maxima and the virtual roughness maxima of this experiment is 


\begin{tabular}{|c|c|c|c|c|}
\hline Probe Radius [mm] & 2nd Order Curv. & Predicted Max. [mm] & 2nd Order Max. [mm] & 3rd Order Max [mm] \\
\hline 0.25 & -1.59 & 1.31 & 1.69 & 1.35 \\
0.50 & -1.50 & 1.56 & 1.93 & 1.86 \\
1.00 & -1.23 & 2.06 & 2.28 & 2.57 \\
1.50 & -0.92 & 2.56 & 2.77 & 2.93 \\
\hline
\end{tabular}

TABLE 5

Maxima of DCT roughness estimates based on 2nd and 3rd order fits in a log-log plot (antilog values shown). Curvature of the 2nd order fit on a log-log plot of roughness versus spacing is shown. Predicted maxima from the geometric model are also shown.

$-0.02 \mathrm{~mm}$. The mean absolute difference is $0.16 \mathrm{~mm}$. The absolute curvature of $\Psi(I)$ was found to steadily and significantly decrease with probe size (Table 5). Curvature is a measure of the sensitivity of roughness perception to changes in spacing. Larger absolute curvatures imply large changes in subjective roughness occur for a fixed range of spacings. Thus, as probe size increases sensitivity of subjective roughness to element spacing decreases. The reasons for this effect may be related to the smoother path followed by the larger spherical probes over the texture elements. Real DCTs have not been found to demonstrate this monotonic trend, although absolute curvature for larger probes $(1.5 \mathrm{~mm}$ radius, curvature $=1.08$ ) has been found to be smaller than curvature for smaller ones $(0.5 \mathrm{~mm}$ radius, curvature $=1.31)$ [28]. The average absolute difference between real (determined from Klatzky et al.'s HV data) and virtual texture $\Psi(I)$ curvatures is 0.26 . The results of our experiment thus demonstrate that near equivalence between virtual and real roughness perception can be achieved using a MLHD and an algorithm which accounts for probetexture geometric interaction.

\section{General Discussion}

One of the motivating factors for this study was the previous finding of substantial differences in roughness perception for virtual vs. real textures [4], [7], [9]. Kornbrot et al. found that the psychophysical function for virtual texture roughness, $\Psi(I)$, was linear and varied substantially between subjects [4]. They hypothesized that the analytic combination of highly variable subject responses, with subjects having both negatively and positively sloped $\Psi(I)$ s, produced the inverted " $\mathrm{U}$ "shaped function seen by Klatzky et al. in studies of real texture [28]. Noting that Kornbrot's group used a virtual

\begin{tabular}{|c|c|c|c|}
\hline Factor & DOF & F & p-value \\
\hline Curvature & $3 / 92$ & 3.06 & $<0.05$ \\
Maximum Roughness & $3 / 92$ & 0.21 & $>0.05$ \\
2nd Order Maxima & $3 / 92$ & 25.39 & $<0.0001$ \\
3rd Order Maxima & $3 / 92$ & 22.61 & $<0.0001$ \\
\hline
\end{tabular}

TABLE 6

Significance of probe size for DCTs, determined with a 1-way ANOVA on curvature, maximum roughness (curve height) and maxima of 2 nd and $3 r d$ order fits to roughness estimates.

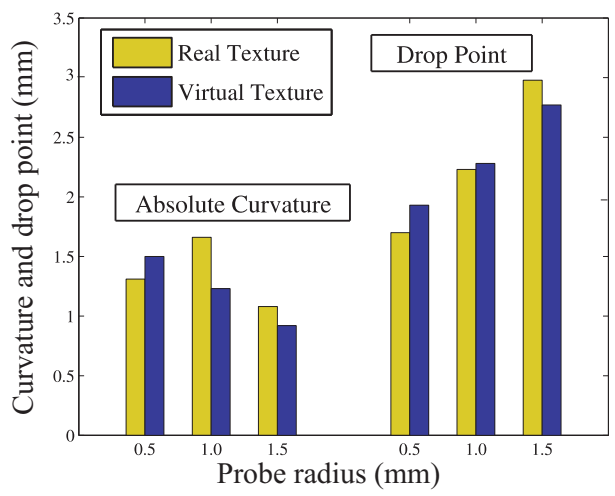

Fig. 13. Comparison of the curvature and maxima location (DPs) of the psychophysical function for roughness $(\Psi(I))$ for real and virtual spherical probes on DCTs. Real data are taken from Klatzky et al. [28].

point-probe on SGTs for their experiments while Klatzky et al.'s studies used a spherical probe on DCTs, we hypothesized that the discrepancies are related to probetexture geometry, rather than some inherent difference between virtual and real textures or in data analysis.

Our first experiment explored the use of a point-probe on SGTs using the MLHD in a manner similar to Kornbrot et al.'s studies [4]. This provided a baseline against which to compare previous studies of virtual texture. We found the psychophysical roughness function for a point-probe on SGTs to be neither linear nor " $U$ "shaped. Instead it consisted of a long initial plateau of high roughness, followed by a linear decline. The slope of the declining portion was -0.82 , virtually identical to that found by Kornbrot et al. While we observed some variation between subjects, the standard error of the function's slope was only 0.13 . It seems clear that, for a virtual point-probe on SGTs, a linear $\Psi(I)$ is produced for large element spacings.

It should be noted that the position bandwidth and maximum compliance of the haptic device used to generate textures may affect these results. Since the MLHD has a position bandwidth of approximately $120 \mathrm{~Hz}$, a probe speed of 30-60 mm/sec, which is typical of the subjects in our experiments, will significantly attenuate the motion of the probe for spacings smaller than $0.2 \mathrm{~mm}$. For these speeds, the PHANToM ${ }^{\mathrm{TM}}$ device, used by Kornbrot's group, with its position bandwidth of $50 \mathrm{~Hz}$ [35], will not produce reliable textures for spacings smaller than approximately $0.5 \mathrm{~mm}$. A second issue related to the 
PHANToM ${ }^{\mathrm{TM}}$ is its maximum stiffness of approximately $2 \mathrm{~N} / \mathrm{mm}$. Such a low stiffness (high compliance) results in a $\Psi(I)$ which shows low magnitude estimates and is nearly linear and highly variable [12]. This suggests that the inter-subject variability seen by Kornbrot may reflect hardware limitations.

The two initial experiments, SGT with point probe and TGT with spherical probe, produced markedly different psychophysical functions. In contrast to the plateau and linear decrease found for the SGT, when a TGT was explored with a spherical probe, the shape of the psychophysical roughness function approximated a step (see Figure 7). These differences reflect the nature of the interaction between probe and surface, as follows. When an SGT is explored with a point-probe, the probe is able to fully traverse the SGT, passing through the full amplitude of the sinusoid, regardless of spacing. Its vertical motion is thus unaffected by element spacing. The slope of the sinusoid decreases with increasing period, however, and thus the rate of change of position and force should decrease (for large unattenuated periods). If the rate of change of position or force are the salient physical properties of roughness perception, then, for SGTs explored with a point-probe, roughness should start near a maximum and decrease as period increases. In contrast, when a TGT is explored with a spherical probe, since the side angle of the trapezoid in the TGT is constant, element spacing will not affect the rate of change of probe position or force. As spacing increases, however, elements are encountered less frequently. If the time-averaged change in position or force underlies perceived roughness, a decline from peak roughness could be expected, its magnitude depending on the amount of time or space used by the perceptual system to integrate the input signal.

The TGT experiment also demonstrates that changing probe size shifts the onset of maximum roughness to larger element spacings. Whereas the quadratic roughness function seen by Klatzky et al. for real textures is not observed, the onset of maximum roughness for TGTs can be considered to be equivalent to Klatzky et al.'s DPs and is predicted by the earlier geometric model with reasonable accuracy (see Table 3). The geometric model consistently underestimates the onset of maximum roughness, however. This is likely due to its quasistatic nature. A simple modification, which takes into account the velocity of the probe tip across the surface, improves consistency between the model and the experimental DP data (see Figure 8). Whereas the DPs, even with the revised model, still do not precisely match experimental findings for TGTs, the trend of increasing DP with increasing probe radius is significant by statistical measurement.

In the third experiment, when the CS algorithm was used to model the interaction of a spherical probe with DCTs, the data matched judgments of comparable real textures [28] to a high degree of accuracy. Nearly identical DPs were obtained at each probe radius, and the curvatures of the $\Psi(I) \mathrm{s}$ were very similar. Data from virtual and real textures also corresponded with respect to the tendency for the DP to increase, and curvature to decrease, with increasing probe size. The decrease in $\Psi(I)$ curvature seen with increased probe size can be related to the path the probe follows as it traverses the constraint surface. For spherical probes, the radius of the arc of motion that occurs while crossing an element lip is related to the radius of the probe itself. A larger probe will follow a path with more gradual changes in $z$-axis motion and forces, than will a smaller one (see Figure 14). If the physical property experienced as roughness relates to the rate of change in position or force, the maximum perceived roughness for larger probes may be decreased (as seen in Table 5). More gradual changes in motion and force also require larger element spacings to induce significant changes in roughness. This effectively lowers subjective sensitivity to changes in element spacing, reducing the $\Psi(I)$ curvature.

Differences in $\Psi(I)$ between the TGTs and DCTs are likely attributable to the regularity of the TGTs. When a regular grating is crossed by a spherical probe, roughness increases with increasing penetration depth, as predicted by the Klatzky et al. geometric model. Maximum penetration produces maximum roughness at roughly the predicted DP. The same factors govern roughness for DCTs; however, once maximum penetration has occurred, the amount of free space between DCT elements increases much more rapidly than for TGTs. For DCTs, therefore, the probe will encounter elements much less frequently as element spacing increases. Thus roughness diminishes more rapidly after reaching the maximum for DCTs than it does for TGTs.

The studies reported here lead to the conclusion that with careful simulation of the geometry of probe-texture interaction using a high-fidelity haptic device, virtual roughness perception is essentially equivalent to real roughness perception. The shape of the psychophysical function for roughness clearly reflects probe-texture geometry, rather than hardware capabilities or some fundamental difference between virtual and real texture perception. Further work on this project will be directed at elucidating the physical variables that reflect the probe-texture interaction and thereby give rise to the percept of textural roughness.

\section{APPENDIX A \\ Constraint Surface Algorithm}

The constraint surface algorithm describes the motion of a spherical probe tip across a set of elements. The equations below apply to truncated cones but can be also used for trapezoidal gratings by substituting grating top and bottom widths for cone base and top radii.

As seen in Figure 14, each element has a base radius $R_{\text {base }}$, and a top radius $R_{\text {top }}$. The sides of the cone rise with angle $\alpha$, to a circular plateau. The height of the cone, $C_{h}$, is determined by these parameters as 


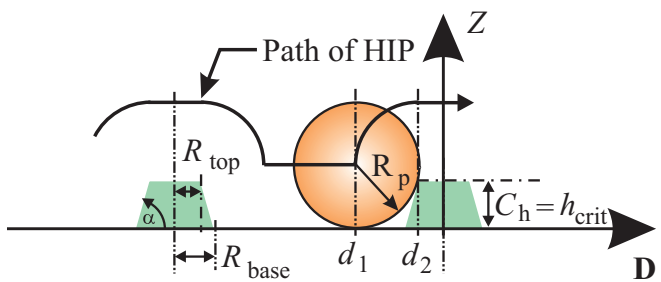

(a)

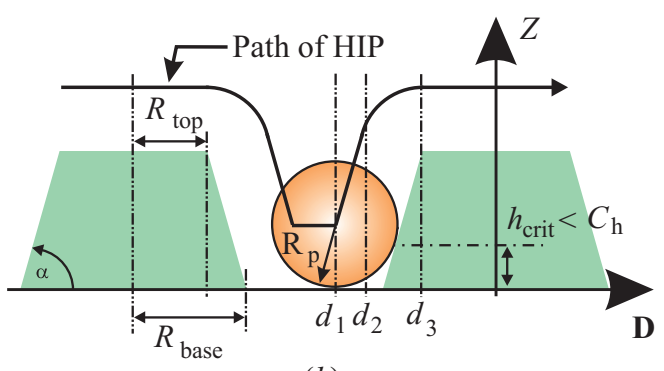

(b)

Fig. 14. (a) Large spherical probe moving over a smaller cone where first contact occurs at the cone's upper lip, (b) smaller spherical probe moving over a larger cone where first contact occurs below the cone's upper lip.

$$
C_{h}=\left(R_{\text {base }}-R_{\text {top }}\right) \tan (\alpha)
$$

The elements are situated on a smooth surface defined by the $(x, y)$-plane. The path that a spherical probe will take as it passes over a cone is dictated by the geometry of the probe-element interaction. If a probe of radius $R_{p}$ travels around a convex corner that has greater curvature than the sphere itself, it moves along an arc with radius $R_{p}$. Otherwise, it moves a distance $R_{p}$ from the surface and parallel to it. The probe path is therefore governed by a set of piece-wise continuous functions. The inflection points between the functions are determined by the radius of the probe and the shape of the cone.

In determining the probe path, it is first necessary to locate the probe with respect to the nearest cone element. The center of the probe is mapped onto the HIP. The cartesian distance in the $(x, y)$-plane, $d$, is found from the center of the probe to the center of the nearest cone. If the probe is farther than $d_{1}$, the point of initial contact, from any cone, the height of the HIP above the surface, $z_{\text {des }}$, will simply be that of the probe radius itself. The location of $d_{1}$, and other points of inflection in the probe path, are determined from the probe radius and cone side angle and are defined as distances from the nearest cone element center.

A spherical probe may either make first contact with a cone at its upper edge as shown in Fig. 14a or at the point along the cone's leading edge where the tangent to the sphere's surface is equal to $\alpha$ as seen in Fig. 14b. This critical height of the first contact, $h_{\text {crit }}$, is found as

$$
h_{\text {crit }}=R_{p}(1-\cos (\alpha)) \text {, }
$$

and is used to divide probe-cone contacts into two cases. The first case (Fig. 14a), in which $C_{h} \leq h_{\text {crit }}$, has two probe path inflection points, $d_{1}$ and $d_{2}$, which are found as:

$$
d_{1}=R_{\text {top }}+\sqrt{C_{h}\left(2 R_{p}-C_{h}\right)}
$$

$$
d_{2}=R_{\text {top }}
$$

In this case, the probe's distance, $d$, from the center of the nearest cone dictates its height, $z_{\text {des }}$, above the $(x, y)$-plane as follows:

$$
\begin{gathered}
d \geq d_{1}: z_{\text {des }}=R_{p}, \\
d_{2} \leq d<d_{1}: z_{\text {des }}=C_{h}+\sqrt{R_{p}^{2}-\left(d-R_{\text {top }}\right)^{2}}, \\
0 \leq d<d_{2}: z_{\text {des }}=C_{h}+R_{p} .
\end{gathered}
$$

The second case, in which $C_{h} \geq h_{\text {crit }}$ (Fig. 14b), has three probe path inflection points

$$
\begin{gathered}
d_{1}=R_{\text {base }}+R_{p} \sin (\alpha)-h_{\text {crit }} \cot (\alpha), \\
d_{2}=R_{\text {top }}+R_{p} \sin (\alpha), \\
d_{3}=R_{\text {top }} .
\end{gathered}
$$

In this case, the probe's height, $z_{\text {des }}$, above the $x, y$ plane is determined by $d$ as:

$$
\begin{gathered}
d \geq d_{1}: z_{\text {des }}=R_{p}, \\
d_{2} \leq d<d_{1}: z_{\text {des }}=\frac{R_{p}+\left(R_{\text {base }}-d\right) \sin (\alpha)}{\cos (\alpha)}, \\
d_{3} \leq d<d_{2}: z_{\text {des }}=C_{h}+\sqrt{R_{p}^{2}-\left(d-R_{\text {top }}\right)^{2}}, \\
0 \leq d<d_{3}: z_{\text {des }}=C_{h}+R_{p} .
\end{gathered}
$$

\section{ACKNOWLEDGMENTS}

The authors would like to thank the National Science Foundation for its support under grants IRI-9420869, IIS9802191, EIA-0321057, and IIS-0413085.

\section{REFERENCES}

[1] C. J. Cascio and K. Sathian, "Temporal cues contribute to tactile perception of roughness," The Journal of Neuroscience, vol. 21, no. 14, pp. 5289-5296, July 2001.

[2] C. E. Connor and K. O. Johnson, "Neural coding of tactile texture: Comparison of spatial and temporal mechanisms for roughness perception," Journal of Neuroscience, vol. 12, no. 9, pp. 3414-3426, September 1992.

[3] R. Klatzky, S. Lederman, C. Hamilton, and G. Ramsay, "Perceiving roughness via a rigid probe: Effects of exploration speed," in DSC-Vol 67, Proceedings of the ASME Dynamic Systems and Control Division-1999, 1999, pp. 27-33.

[4] D. Kornbrot, P. Penn, H. Petrie, S. Furner, and A. Hardwick, "Roughness perception in haptic virtual reality for sighted and blind people," Perception and Psychophysics, vol. 69, no. 4, pp. 502512, 2007.

[5] M. Lawrence, R. Kitada, R. Klatzky, and S. Lederman, "Haptic roughness perception of linear gratings via bare finger or rigid probe," Perception, vol. 36, pp. 547-557, 2007. 
[6] S. J. Lederman, "Tactile roughness of grooved surfaces: The touching process and effects of macro- and microsurface structure," Perception and Psychophysics, vol. 16, no. 2, pp. 385-395, 1974.

[7] M. R. McGee, P. Gray, and S. Brewster, "Haptic perception of virtual roughness," in CHI 2001, March-April 2001, pp. 155-156.

[8] E. M. Meftah, L. Belingard, and C. E. Chapman, "Relative effects of the spatial and temporal characteristics of scanned surfaces on human perception of tactile roughness using passive touch," Experimental Brain Research, vol. 132, pp. 351-361, 2000.

[9] P. Penn, D. Kornbrot, S. Furner, A. Hardwick, C. Colwell, and H. Petrie, "Roughness perception in haptic virtual reality: The impact of the haptic device, endpoint and visual status," 2004, unpublished manuscript.

[10] A. M. Smith, C. E. Chapman, M. Deslandes, J.-S. Langlais, and M.-P. Thibodeau, "Role of friction and tangential force variation in the subjective scaling of tactile roughness," Experimental Brain Research, vol. 144, pp. 211-223, 2002.

[11] M. Taylor and S. Lederman, "Tactile roughness of grooved surfaces: A model and the effect of friction," Perception and Psychophysics, vol. 17, no. 1, pp. 23-36, 1975.

[12] B. Unger, R. Hollis, and R. Klatzky, "The geometric model for perceived roughness applies to virtual textures," in IEEE Symposium on haptic interfaces for virtual environment and teleoperator systems, 2008, March 2008, pp. 3-10.

[13] S. J. Lederman and M. M. Taylor, "Fingertip force, surface geometry, and the perception of roughness by active touch." Perception and Psychophysics, vol. 12, pp. 401-408, 1972.

[14] S. Lederman, J. Loomis, and D. Williams, "The role of vibration in the tactual perception of roughness," Perception and Psychophysics, vol. 32, no. 2, pp. 109-116, 1982.

[15] M. Taylor, S. Lederman, and R. Gibson, Handbook of Perception. New York: Academic Press, 1973, vol. 3, ch. 12, pp. 251-272.

[16] R. LaMotte and V. Mountcastle, "Capacities of humans and monkeys to discriminate vibratory stimuli of different frequency and amplitude: A correlation between neural events and psychological measurements," Journal of Neurophysiology, vol. 38, no. 3, pp. 53959, May 1975.

[17] I. Darian-Smith, I. Davidson, and K. O. Johnson, “Peripheral neural representation of the spatial dimensions of a textured surface moving across the monkey's finger pad," Journal of Physiology, vol. 309, pp. 135-146, 1980.

[18] I. Darian-Smith and L. E. Oke, "Peripheral neural representation of the spatial frequency of a grating moving across the monkey's finger pad," Journal of Physiology, vol. 309, pp. 117-133, 1980.

[19] G. D. Lamb, "Tactile discrimination of textured surfaces: Peripheral neural coding in the monkey," Journal of Physiology, vol. 338, pp. 567-587, 1983.

[20] , "Tactile discrimination of textured surfaces: Psychophysical performance measurements in humans," Journal of Physiology, vol. 338, pp. 551-565, 1983.

[21] E. Gamzu and E. Ahissar, "Importance of temporal cues for tactile spatial-frequency discrimination," The Journal of Neuroscience, vol. 21, no. 18, pp. 7416-7427, September 2001.

[22] G. A. Gescheider, S. J. Bolanowski, T. C. Greenfield, and K. E. Brunette, "Perception of the tactile texture of raised-dot patterns: A multidimensional analysis," Somatosensory and Motor Research, vol. 22, no. 3, pp. 127-140, September 2005.

[23] M. Hollins, F. Lorenz, and D. Harper, "Somatosensory coding of roughness: The effect of texture adaptation in direct and indirect touch," The Journal of Neuroscience, vol. 26, no. 20, pp. 5582-5588, May 2006.

[24] M. Hollins, S. J. Bensmaïa, and S. Washburn, "Vibrotactile adaptation impairs discrimination of fine, but not coarse, textures," Somatosensory and Motor Research, vol. 18, no. 4, pp. 253-262, 2001.

[25] M. Hollins and S. R. Risner, "Evidence for the duplex theory of tactile texture perception," Perception and Psychophysics, vol. 62, no. 4, pp. 695-705, 2000.

[26] R. L. Klatzky and S. J. Lederman, "Tactile roughness perception with a rigid link interposed between skin and surface," Perception and Psychophysics, vol. 61, no. 4, pp. 591-607, 1999.

[27] S. Lederman and R. Klatzky, "Sensing and displaying spatially distributed fingertip force in haptic interfaces for teleoperator and virtual environment systems," Presence, vol. 8, no. 1, pp. 86-103, February 1999

[28] R. L. Klatzky, S. J. Lederman, C. Hamilton, M. Grindley, and R. H. Swendsen, "Feeling textures through a probe: Effects of probe and surface geometry and exploratory factors," Perception and Psychophysics, vol. 65, no. 4, pp. 613-631, 2003.

[29] S. A. Wall and W. S. Harwin, "Effect of physical bandwidth on perception of virtual gratings," in Proceedings of the ASME Dynamic Systems and Control Division (Symposium on Haptic Interfaces for Virtual Environments and Teleoperators), ser. The Winter Annual Meeting of ASMEDSC, vol. 69, no. 2, 2000, pp. 1033-1039.

[30] R. Hollis, S. Salcudean, and A. P. Allan, "A six-degree-of-freedom magnetically levitated variable compliance fine motion wrist: Design, modeling, and control," IEEE Transactions on Robotics and Automation, vol. 7, no. 3, pp. 319-332, June 1991.

[31] P. Berkelman and R. Hollis, "Lorentz magnetic levitation for haptic interaction: Device design, performance, and integration with physical simulations," The International Journal of Robotics Research, vol. 19, no. 7, pp. 644-667, July 2000.

[32] P. J. Berkelman, "Tool-based haptic interaction with dynamic physical simulations using Lorentz magnetic levitation," Ph.D. dissertation, Carnegie Mellon University, The Robotics Institute, 1999.

[33] K. Muck, "Motion planning in constraint space," Robotics and Automation, 1988. Proceedings., 1988 IEEE International Conference on, pp. 633-635 vol.1, 24-29 Apr 1988.

[34] D. T. Blake, S. Hsiao, and K. O. Johnson, "Neural coding mechanisms in tactile pattern recognition: The relative contributions of slowly and rapidly adapting mechanoreceptors to perceived roughness," The Journal of Neuroscience, vol. 17, no. 19, pp. 7480$7489,1997$.

[35] M. C. Cavusoglu, D. Feygin, and F. Tendick, "A critical study of the mechanical and electrical properties of the PHANToM haptic interface and improvements for high-performance control," Presence: Teleoperators and Virtual Environments, vol. 11, no. 6, pp. $555-568,2002$.

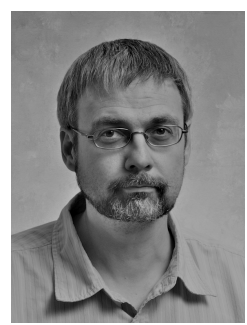

Bertram Unger (MD University of Manitoba, PhD Carnegie Mellon University, Robotics) is Assistant Professor and Research Director of the Clinical Learning and Simulation Facility in the Faculty of Medicine at the University of Manitoba. He is also a clinician with the University's Department of Internal Medicine, Section of Critical Care and has an adjunct appointment with its Faculty of Engineering. $\mathrm{He}$ recently completed a post-doctoral Fellowship at the University of Pittsburgh in the Faculty of

Bioengineering.

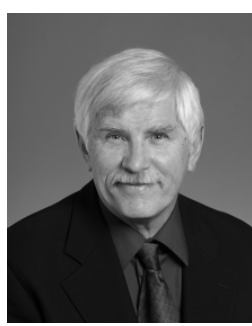

Ralph Hollis (PhD University of Colorado, Physics) is Research Professor of Robotics and Electrical and Computer Engineering at Carnegie Mellon University. Dr. Hollis was a Research Staff Member at the Thomas J. Watson Research Center from 1978-93 where he worked in magnetism, acoustics, and robotics, and was Manager of Advanced Robotics from 1986-93. He is a member of the American Physical Society and a Fellow of IEEE. He is founding director of the Microdynamic Systems Laboratory at Carnegie Mellon University where his research centers on haptics, agile manufacturing, and dynamically-stable mobile robots.

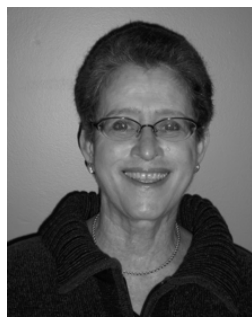

Roberta Klatzky (PhD Stanford, Psychology) is Professor of Psychology and Human Computer Interaction at Carnegie Mellon University. Her research interests are in human perception and cognition, with special emphasis on spatial cognition and haptic perception. She has done extensive research on human haptic and visual object recognition, navigation under visual and nonvisual guidance, and perceptually guided action, with application to navigation aids for the blind, haptic interfaces, exploratory robotics, image-guided surgery and virtual environments. 